NEUROLOGICAL PICTURE

\title{
Spontaneous occipital subdural haematoma
}

A case of spontaneous occipital subdural haematoma due to ruptured aneurysm is described. A 70 year old woman was admitted on 20 July 2000, because of spontaneous severe occipitalgia. She had no neurological deficits. Several years before she had had a similar episode of severe headache, but she had not sought medical attention. Initial
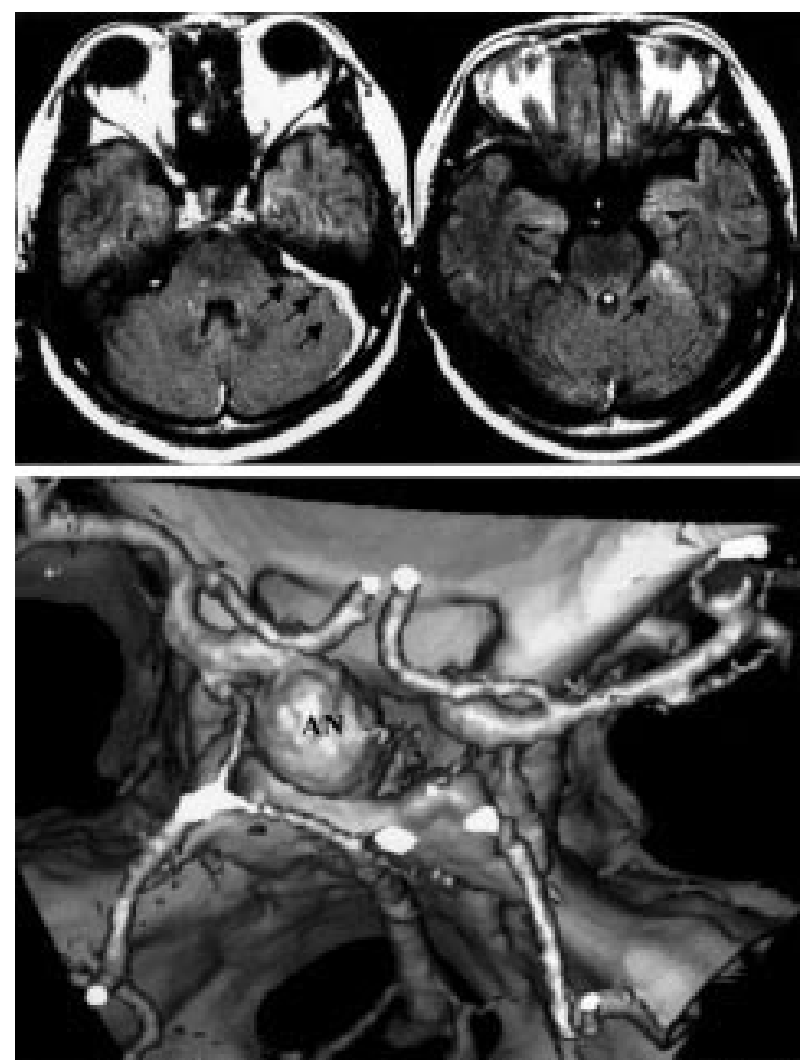

Figure 1 (Upper) Axial fluid attenuated inversion recovery MRI showing an occipital subdural haematoma (upper left) and absence of subarachnoid haemorrhage in the basal cistern (upper right). Arrows indicating subdural haematoma. (Lower) Three dimensional CT angiogram showing a large left internal carotid artery aneurysm extending posteromedially. AN=aneurysm.
CT on admission showed a subtentorial high density area indicating a subdural haematoma. Brain MRI showed an occipital subtentorial subdural haematoma, successive flow void indicating an internal carotid artery aneurysm in the basal cistern, but no subarachnoid haemorrhage (fig 1 upper). Three dimensional CT angiography disclosed a large left internal carotid artery aneurysm extending posteromedially (fig 1 lower), and no abnormal vessels such as arteriovenous malformation in the occipital fossa and cervical junction at other sites. She underwent standard pterional craniotomy the next day. The carotid cistern was opened to release CSF. No subarachnoid haemorrhage was found in the basal cistern. The proximal portion of the internal carotid artery was exposed by partial elimination of the anterior clinoid process. The neck of the aneurysm was clipped using a neuroendoscope. The aneurysm arose from the medial wall of the internal carotid artery between the ophthalmic artery and the posterior communicating artery and projected to the occipital fossa. The body of the aneurysm had adhered to the arachnoid membrane under the tentorium. She made an uneventful recovery and returned to her previous life.

The clinical incidence of subdural haematoma complicated with subarachnoid haemorrhage due to ruptured aneurysm is $0.5 \%-7.9 \%$ of all cases of SAH. Subdural haematoma occurring without subarachnoid haemorrhage due to ruptured aneurysm is extremely rare. ${ }^{1}$ The probable mechanism of subdural haematoma in our patient was the presence of adhesions between the aneurysm and the arachnoid membrane due to previous minor haemorrhages. ${ }^{12}$ Acute spontaneous occipital subdural haematoma may originate from a ruptured internal carotid artery aneurysm.

A UTSUNOMIYA N NARITA

Department of Neurosurgery, Yonezawa City Hospital, 6-36 Aioi-cho, Yonezawa, Yamagata 992-8502, Japan

Correspondence to: Dr A Utsunomiya sy4a-utnm@asahi-net.or.jp

1 O'Sullivan MG, Whyman M, Steers JW, et al. Acute subdural haematoma secondary to ruptured intracranial aneurysm: diagnosis and management. Br F Neurosurg 1994;8:439-45.

2 Kamiya $\mathrm{K}$, Inagawa T, Yamamoto $\mathrm{M}$, et al. Subdural hematoma due to ruptured intracranial aneurysm. Neurol Med Chir (Tokyo) 1991;31:82-6. 